\title{
Implication des virus dans la sclérose en plaques
}

La sclérose en plaques est une maladie inflammatoire du système nerveux central, résultat d'une attaque immunitaire suivie d'une destruction de la gaine de myéline qui enrobe normalement les fibres nerveuses. Bien que la cause de la maladie nous échappe toujours, il est clair qu'elle implique à la fois une susceptibilité génétique et des facteurs liés à l'environnement, probablement infectieux. Une quinzaine de virus ont été associés à la sclérose en plaques, sans qu'un candidat plus convaincant que les autres s'impose. Les coronavirus sont parmi les derniers en liste, fournissant depuis plusieurs années chez la souris l'un des modèles les plus intéressants d'une maladie virale rassemblant plusieurs des caractéristiques de la sclérose en plaques. L'hypothèse virale de la maladie demeure raisonnable mais il est peu probable qu'un seul virus soit en cause. En effet, plusieurs virus pourraient déclencher la maladie par un mécanisme commun d'induction de réactions auto-immunitaires chez des

\section{Pierre Talbot}

P. Talbot: docteur en biochimie, professeur-chercheur. Laboratoire de neuro-immunologie, centre de recherche en virologie, Institut Armand-Frappier, Université du Québec, 531, boulevard des Prairies, case postale 100, succursale Laval-des Rapides, Laval, Québec, H7N 4 Z3 Canada.
} personnes génétiquement susceptibles. Les programmes de vaccination contre la rougeole, la rubéole et les oreillons feront-ils diminuer l'incidence de la sclérose en plaques?

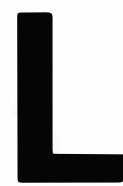

a sclérose en plaques est la maladie du système nerveux central la plus fréquente chez les jeunes adultes dans les régions touchées, dont le Canada et le nord de l'Europe. Environ 50000 Canadiens en sont affectés, soit près d'une personne sur 500 . La maladie touche les jeunes adultes, surtout entre 20 et 50 ans, avec un pic vers l'âge de 30 ans. Décrite pour la première fois il y a cent vingt-cing ans par Jean-Martin Charcot, la sclérose en plaques se manif este par une inflammation du système nerveux central qui détruit la gaine de myéline des fibres nerveuses. Cela interrompt et distord le flux naturel des impulsions nerveuses, provoquant divers symptômes: problèmes d'acui- té visuelle, engourdissements, pertes d'équilibre, fatigue extrême, tremblements et même paralysie. Quoique les malades puissent jouir de phases de rémission de quelques mois ou même quelques années, la sclérose en plaques est le plus souvent progressive, des attaques ou exacerbations imprévisibles contribuant à aggraver l'invalidité du malade. Le diagnostic initial de la maladie, longtemps délicat, est facilité aujourd'hui par l'imagerie par résonance magnétique, qui permet d'observer de façon non agressive des coupes du cerveau et d'y détecter les zones touchées, simplement en baignant le patient dans un champ magnétique et en lui envoyant une onde radio de fréquence connue. 


\section{RÉFÉRENCES}

1. Sadovnick AD, Ebers GC. Epidemiology of multiple sclerosis: a critical overview. Can J Neurol Sci 1993; 20: 17-29.

2. Oger J. Génétique de la sclérose en plaques. médecine/sciences 1987; 3: 13-8.

3. Hillert J, Olerup O. Multiple sclerosis is associated with genes within or close to the HLA-DR-DQ subregion on a normal DR15,DQ6,Dw2 haplotype. Neurology 1993; 43: $163-8$.

4. Duquette P, Pleines J, Girard M, Charest L, Senecal-Quévillon M, Masse C. The increased susceptibility of women to multiple scle rosis. Can J Neurol Sci 1992; 19 466-71.

5. Steinman L, Oksenberg JR, Bernard CCA. Association of susceptibility to multiple sclerosis with TCR genes. Immunol Today 1992; 13: 49-51.

6. Utz U, Biddison WE, Mc Farland HF McFarlin DE, Flerlage M, Martin R. Skewed $\mathrm{T}$-cell receptor repertoire in genetically identical twins correlates with multiple sclerosis. Nature 1993; 364: 243-6.

7. Skegg DCG. Multiple sclerosis. Nature or nurture. Br Med J 1991 ; 302 : 247-8.

8. Talbot P, Jouvenne P. Le potentiel neurotrope des coronavirus. medecine/sciences 1992; 8 : 119-25.

9. Weiner LP. Pathogenesis of demyelination induced by mouse hepatitis virus (JHM virus). Arch Neurol 1973; 28: 298-303.

10. Lampert PW, Sims JK, Kniazeff AJ. Mechanism of demyelination in JHM virus encephalomyelitis. Acta Neuropathol 1973; 24: 76-85.

11. Wang FI, Stohlman SA, Fleming JO. Demyelination induced by murine hepatitis virus JHM strain (MHV-4) is immunologically mediated. J Neuroimmunol 1990; 30:3141 .

12. Watanabe $\mathrm{R}$, Wege $\mathrm{H}$, ter Meulen V. Adoptive transfer of EAE-like lesions from rats with coronavirus-induced demyelinating encephalomyelitis. Nature 1983; 305
Malgré d'intenses efforts de recherche clinique et fondamentale, la sclérose en plaques demeure toujours de cause inconnue. Cette maladie neurologique semble résulter d'une activation incontrôlée et inexpliquée du système immunitaire qui attaque le système nerveux central (cerveau et moelle épinière). Plusieurs études épidémiologiques [1] nous permettent de conclure que certains facteurs génétiques prédisposent à la maladie mais que des facteurs environnementaux sont aussi impliqués. La sclérose en plaques est donc une maladie d'étiologie multifactorielle, ce qui rend très difficile la recherche des causes spécifiques et de nouvelles approches thérapeutiques.

\section{Facteurs génétiques de susceptibilité}

La susceptibilité à la sclérose en plaques est liée à plusieurs facteurs génétiques [2]. Chez les habitants de souche caucasienne de l'Europe et de l'Amérique du Nord, la seule association génétique bien documentée de la maladie est avec les antigènes d'histocompatibilité de classe II de type HLA-DR2 dont les gènes sont portés par le chromosome 6 [3]. Récemment, la prépondérance féminine de la sclérose en plaques (2 à 3 fois plus de maladie chez les femmes) a même été corrélée à la présence plus fréquente de l'allèle HLA-DR2 chez les femmes, ce qui n'exclut pas la participation de facteurs hormonaux dont on connaît l'influence sur les réponses immunitaires et qui pourraient contribuer à expliquer la répartition inégale selon les sexes de plusieurs maladies autoimmunes [4].

Les antigènes d'histocompatibilité de classe II, comme les HLA-DR chez l'homme, sont impliqués dans la présentation d'antigènes au système immunitaire. Il s'agit en général d'antigènes étrangers, comme les protéines virales ou bactériennes présentes dans l'organisme à la suite d'une maladie infectieuse. En revanche, il est possible que le système immunitaire reconnaisse des antigènes propres à l'organisme, les antigènes du "soi", si la tolérance normalement développée à ceux-ci est rompue ou n'a pu être établie lors du développement de l'individu. Étant donné que la myéline apparaît tardivement dans l'ontogénie et n'est peut-être pas présente dans le thymus, qui semble être l'organe clé impliqué dans l'apparition de la tolérance, le système immunitaire peut ne pas être tolérant à la myéline, même chez les sujets normaux. Cela pourrait expliquer l'antigénicité de la myéline observée chez plusieurs sujets normaux. En revanche, la myéline se situant dans un site que l'on dit immunologiquement privilégié, c'est-à-dire en général non accessible au système immunitaire, cela ne cause normalement pas de problèmes pathologiques.

La présentation des antigènes au lymphocyte $\mathrm{T}$ auxiliaire, le chef d'orchestre de la réponse immunitaire, déclenche ensuite tous les mécanismes spécifiques de protection de l'organisme. Cette présentation implique un complexe trimoléculaire entre l'antigène d'histocompatibilité HLA de classe II, une portion ou fragment peptidique de l'antigène imbriqué dans une région particulière de l'antigène HLA surnommée "niche à peptide" et le récepteur $\mathrm{T}$, une molécule complexe présente à la surface des lymphocytes T. L'hypothèse selon laquelle la susceptibilité à la sclérose en plaques impliquerait aussi les gènes codant pour les récepteurs T $\left(\mathrm{m} / \mathrm{s} n^{\circ} 7\right.$, vol. $6, p$. 711) suscite de très nombreuses recherches dont les résultats demeurent à ce jour controversés [5]. Il est intéressant de noter qu'une étude récente chez des jumeaux monozygotes a démontré une utilisation biaisée de certaines classes de récepteurs $\mathrm{T}$ lors du développement de la sclérose en plaques [6]. De tels travaux laissent pointer une lueur d'espoir dans l'élaboration d'approches thérapeutiques qui pourraient cibler spécifiquement ces lymphocytes $\mathrm{T}$ autoréactifs.

\section{Facteurs environnementaux mis en cause}

Heureusement, toutes les personnes génétiquement prédisposées à développer la sclérose en plaques ne le font pas. La prévalence de la maladie augmente géographiquement selon la latitude au nord et au sud de l'équateur, avec quelques exceptions 
comme l'absence remarquable de la maladie sur des îles au nord et au sud du Japon et certaines régions du nord de l'Europe comme la côte de la Norvège. Quelques études, qui ont évalué le développement de la sclérose en plaques dans des populations ayant migré entre des régions où la prévalence de la maladie est différente, ont montré que l'exposition à un facteur environnemental avant l'âge de 15 ans [7] est nécessaire au déclenchement de la maladie. Enfin, l'apparition soudaine de la sclérose en plaques dans les îles Feroe, qui en étaient auparavant exemptes, à la suite du débarquement des troupes britanniques en 1940 (46 cas identifiés entre 1943 et 1982 sur une population d'environ 45000 individus) suggère une nature épidémique possible de la maladie; elle indique aussi qu'une exposition d'au moins deux ans à l'agent infectieux probablement introduit à cet endroit par les Britanniques a été nécessaire et que les signes cliniques de la maladie ne sont apparus qu'après une moyenne de six années d'incubation présumée.

Ces observations décrivant l'influence de l'environnement sur le développement de la sclérose en plaques pourraient s'expliquer par la contribution d'un agent infectieux, la prévalence de la maladie étant alors liée aux facteurs qui affectent l'infection, comme le climat ou l'hygiène. A cet effet, il est fascinant de noter que la rougeole, une maladie virale, apparaît en général plus tôt dans la vie des enfants des régions tropicales et intertropicales, où la sclérose en plaques est rare, que dans les régions tempérées où la maladie est plus fréquente. Par ailleurs, il est bien connu que la paralysie causée par le virus de la poliomyélite est beaucoup plus fréquente et grave chez les personnes qui sont infectées à un âge plus avancé. Il est donc intéressant de spéculer que la sclérose en plaques se développerait chez des individus génétiquement prédisposés et qui contracteraient certaines maladies virales au début de l'adolescence plutôt que dans la petite enfance.

L'hypothèse virale de la sclérose en plaques n'est pas nouvelle puisqu'elle circule depuis plus de cent ans et fait l'objet de programmes actifs de recherche depuis une quarantaine d'années. Elle demeure une $\mathrm{m} / \mathrm{s} n^{\circ} 6$, vol. 11 , juin 95 des plus plausibles, beaucoup plus que certaines suggestions, jamais appuyées sur des données expérimentales convaincantes, impliquant des spirochètes, mycoplasmes, protozoaires, rickettsies et autres microorganismes, ou d'autres facteurs environnementaux tels le climat, le régime, l'exposition à certains animaux et même les rayons cosmiques.

\section{Virus: modèles animaux}

Les modèles animaux de maladies démyélinisantes induites par des virus ont contribué à stimuler l'intérêt pour une possible étiologie virale de la sclérose en plaques; ils ont permis, en outre, de caractériser les diverses étapes de la pathogénie virale expérimentale chez des animaux de laboratoire. Deux modèles retiennent particulièrement l'attention et font appel à la souris et au rat de laboratoire. Il s'agit, d'une part, du virus de l'encéphalomyélite murine de Theiler, un picornavirus de la même famille que le virus de la poliomyélite humaine et, d'autre part, du coronavirus qui cause surtout des maladies respiratoires du type des rhumes chez l'homme. Il sera question ici du deuxième modèle, qui a l'avantage d'avoir une correspondance possible chez l'homme [8].

La maladie neurologique causée chez la souris et le rat parles souches neurotropes du coronavirus murin a fait l'objet de divers travaux depuis une vingtaine d'années. L'infection du système nerveux central de souris adultes par le coronavirus en général entraîne une encéphalomyélite avec infection des neurones et des cellules gliales, astrocytes et oligodendrocytes, qui servent de cellules nourricières et de support dans le système nerveux central. Environ $5 \%$ des animaux survivent à la maladie aiguë et développent alors une maladie neurologique chronique qui affecte la matière blanche et se caractérise par l'apparition de plaques de démyélinisation dans le cerveau et la moelle épinière. Tout comme pour la sclérose en plaques, une remyélinisation et une démyélinisation récurrentes sont observées. L'utilisation de souches virales génétiquement modifiées, ou encore l'injection préalable à l'animal de certains anticorps antiviraux dits «protecteurs", permettent de prévenir l'infection des neurones et d'induire chez tous les animaux infectés une maladie chronique dans laquelle prédomine l'infection des oligodendrocytes.

Le développement de la maladie démyélinisante coronavirale a longtemps été associée à l'infection virale des oligodendrocytes $[9,10]$, ces cellules qui produisent la myéline, une substance qui enrobe les fibres nerveuses et facilite le passage de l'influx nerveux et dont la destruction est responsable des symptômes de la sclérose en plaques. Il a d'ailleurs été démontré dans le modèle animal qu'une infection coronavirale naturelle au niveau des voies nasales peut se propager au cerveau, le virus empruntant le nerf olfactif. Le coronavirus humain étant responsable d'infections du tractus respiratoire supérieur, il est alors tentant de suggérer qu'il pourrait aussi se retrouver dans le système nerveux central et y devenir pathogène chez certaines personnes susceptibles [8].

Des travaux récents suggèrent la possibilité d'un mécanisme immunopathologique de développement de la "sclérose en plaques coronavirale" chez la souris [11], c'est-à-dire une induction par l'infection virale d'une réponse auto-immune ciblant le système nerveux central, tout comme dans la sclérose en plaques. En effet, cette étude démontre qu'il est possible de prévenir la démyélinisation induite par les coronavirus par un traitement immunosuppresseur généralisé et de transférer la maladie auto-immune à des animaux normaux par simple transfert des lymphocytes $T$ de la rate de souris infectées.

Deux autres études démontrent aussi la possibilité que l'infection coronavirale induise une maladie auto-immune. Le transf ert de lymphocytes T des rats infectés par le coronavirus à des animaux naifs induit chez ceux-ci l'encéphalomyélite allergique expérimentale $\left(m / s n^{\circ} 8, v o l .8, p\right.$. 872) [12], une maladie neurologique autoimmune qui constitue la reproduction expérimentale la plus fidèle de la sclérose en plaques. Cette maladie expérimentale est induite par immunisation d'animaux de laboratoire avec la protéine basique de la myéline (myelin basic protein, MBP), une lipoprotéine constituant environ $40 \%$ de la composition moléculaire 


\section{RÉFÉRENCES}

13. Wucherpfennig KW, Weiner HL, Hafler DA. T-cell recognition of myelin basic protein. Immunol Today 1991; 12: 277-82.

14. Kyuwa S, Yamaguchi K, Toyoda Y, Fujiwara $\mathrm{K}$. Induction of self-reactive $\mathrm{T}$-cells after murine coronavirus infection. I Virol 1991 ; 65 : 1789-95.

15. Knobler RL, Haspel MV, Oldstone MBA Mouse hepatitis virus type 4 (JHM strain) induced fatal central nervous system disease. I. Genetic control and the murine neuron as the susceptible site of disease. $J$ Exp Med $1981 ; 153: 832-43$

16. Johnson RC. Viral aspects of multiple sclerosis. In : Koetsier JC, ed. Handbook of clinical neurology: demyelinating diseases, vol. 3: Amsterdam: Elsevier, 1985, 319-36.

17. Salmi A, Reunanen M, Ilonen J. Possible viral etiology of multiple sclerosis. In: Katsuki $\mathrm{S}$, Tsubaki $\mathrm{T}$, Toyokura Y, eds. Interna tional Congress Series, $n^{\circ} 568$, Neurology. Amsterdam: Excerpta Medica, 1981: 41631.

18. Salmi A, Ziola B, Hovi T, Reunanen $M$ Antibodies to coronaviruses $\mathrm{OC} 43$ and $229 \mathrm{E}$ in multiple sclerosis patients. Neurology $1982 ; 32: 292-5$.

19. Goswami KKA, Randall RE, Lange IS, Russell WC. Antibodies against the paramyxovirus SV5 in the cerebrospinal fluids of some multiple sclerosis patients. Nature $1987 ; 327: 2447$

20. Woyciechowska JL, Dambrozia J, Leinikki $P$, et al. Viral antibodies in twins with multiple sclerosis. Neurology $1985 ; 35: 1176-80$.

21. Haase AT, Ventura P, Gibbs CJ, Tourtelotte WW. Measles virus nucleotide sequences: detection by hybridization in situ. Science 1981 ; 212 : 672-4.

22. Cosby SL, McQuaid S, Taylor MJ, Barley M, Rima BK, Martín SJ, Allen IV. Examination of 8 cases of multiple sclerosis and 56 neurological and nonneurological controls for genomic sequences of Measles virus, canine distemper virus, simian virus- 5 and de la myéline et qui semble être une des cibles pathologiques du système immunitaire lors du développement de la sclérose en plaques $\left(\mathrm{m} / \mathrm{s} n^{\circ} 10\right.$, vol. 8, p. 1114). D'ailleurs, une multitude de travaux ont permis d'identifier, sur la séquence en acides aminés de la MBP, des portions encéphalitogènes, c'est-à-dire qui induisent l'encéphalomyélite allergique expérimentale chez diverses espèces animales comme la souris, le rat, le cobaye et le lapin. Chez l'homme, on sait que certaines régions spécifiques de la MBP sont reconnues par les lymphocytes $\mathrm{T}$ autoréactifs de malades atteints de sclérose en plaques, une région de 19 acides aminés étant même considérée immunodominante [13]. Enfin, une étude encore plus récente confirme que l'infection coronavirale provoque l'apparition de lymphocytes T autoréactifs [14].

Une autre ressemblance importante entre les modèles animaux de la sclérose en plaques et la maladie humaine réside dans la détermination de la susceptibilité génétique des animaux de laboratoire à la maladie démyélinisante induite par les virus. Chez la souris, cette susceptibilité implique un gène autosomique dominant situé sur le chromosome 7 [15].
Bien qu'ils soient tous imparfaits, les modèles animaux de la sclérose en plaques nous fournissent des outils précieux pour caractériser les déterminants moléculaires et cellulaires de la maladie et mettre au point des stratégies de prévention et de traitement.

\section{Virus: implication dans la maladie}

Quoique l'étiologie de la sclérose en plaques demeure toujours insaisissable, l'implication d'un ou de plusieurs agent(s) infectieux est largement reconnue, les virus figurant en tête de liste. Au cours des dernières années, divers virus neurotropes ont été considérés comme des candidats plus ou moins sérieux pour la pathogénie de la sclérose en plaques, sans que la communauté scientifique puisse s'entendre sur le candidat le plus probable. Trois approches expérimentales sous-tendent ces hypothèses : isolement de virus à partir de tissus de malades, prépondérance d'anticorps antiviraux chez des malades comparés à des sujets normaux, détection de la présence de virus dans le système nerveux central de malades.

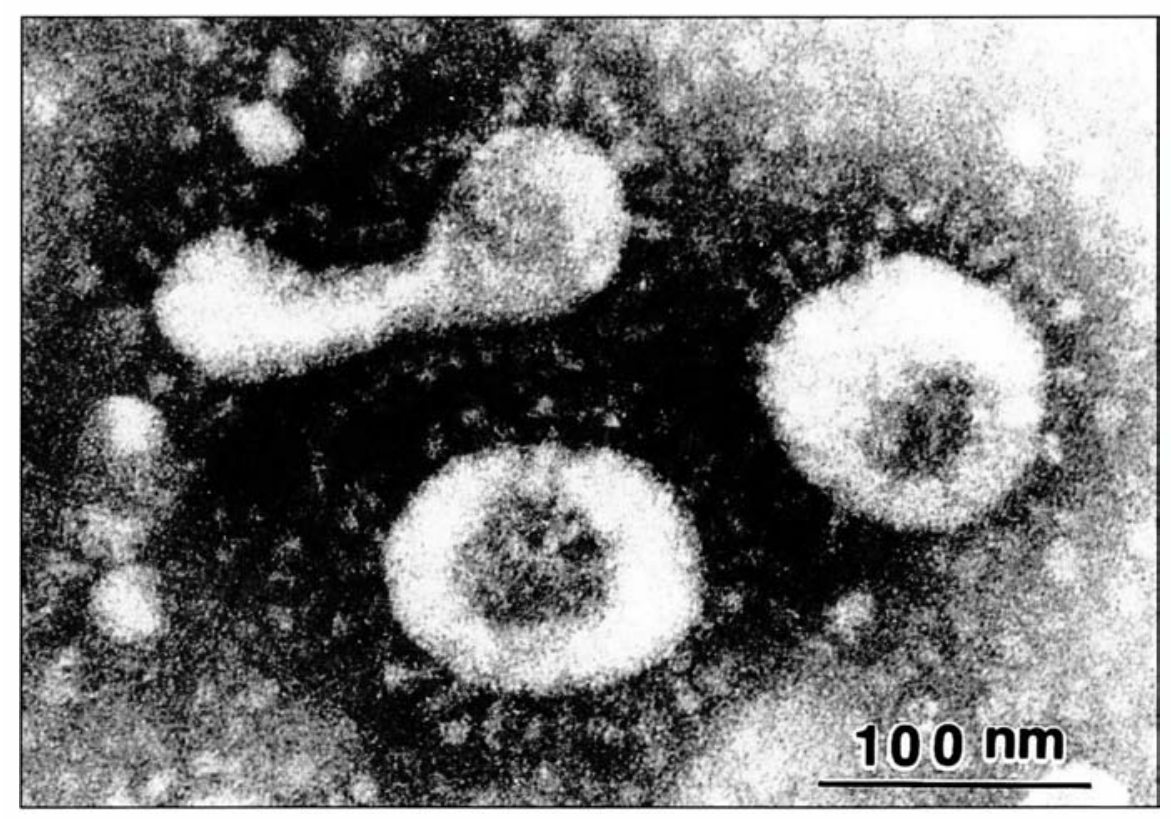

Figure 1. Un des virus associés à la sclérose en plaques: le coronavirus. Coronavirus humain, souche prototype OC43, observé au microscope électronique après coloration négative (préparation de Francine Lambert et microscopie de Robert Alain). 
D'abord, plusieurs études ont fait état de l'isolement de virus à partir de tissus de malades atteints de sclérose en plaques: virus rougeoleux, virus herpes simplex, para-influenzavirus, flavivirus encéphalique transmis par des tiques, virus de la rage, virus simien de type 5, coronavirus et cytomégalovirus [16].

D'autres travaux ont mis en évidence dans le sérum et/ou le liquide céphalo-rachidien de patients atteints de sclérose en plaques une prépondérance significative d'anticorps contre divers virus [17]. Spécifiquement, la présence d'anticorps antiviraux dans le liquide céphalo-rachidien qui baigne le système nerveux central suggère une infection locale. Des études séro-épidémiologiques ont mis en évidence une augmentation significative, chez les patients atteints de sclérose en plaques, d'anticorps contre les virus de la rougeole et herpes simplex, de même que les coronavirus mentionnés plus haut [18], mais aussi contre les virus des oreillons, de la varicelle, de la rubéole, de l'influenza de type $\mathrm{C}$ et de la vaccine (utilisé pour vacciner contre la variole), de même que contre le virus simien de type 5 [19]. Toutefois, la comparaison des anticorps antiviraux entre jumeaux discordants pour la sclérose en plaques, c'est-àdire dont l'un a développé la maladie et l'autre pas, n'a pas indiqué d'augmentation significative d'anticorps contre les virus de la rubéole ou de la vaccine dans le liquide céphalo-rachidien du jumeau atteint [20].

Deux études ont mis en évidence, par une technique d'hybridation in situ (appariement d'une sonde génomique complémentaire au brin d'acide nucléique recherché), la présence du matériel génétique du virus rougeoleux dans le cerveau de quelques patients [21, 22]. Cette même technique a récemment permis de soupçonner la présence de coronavirus chez la moitié des vingt-deux malades testés, des protéines virales étant même détectées par immunohistochimie chez deux d'entre eux [23]. Étrangement, ce coronavirus ressemblait plus aux coronavirus murins qu'aux souches humaines.

Il importe de prendre conscience du fait que l'implication de virus dans le déclenchement de la sclérose en plaques pourrait se faire par l'inter- médiaire d'une infection persistante, au cours de laquelle le virus ne se répliquerait qu'à de très faibles niveaux. D'où l'importance cruciale d'une technologie de pointe pour la détection de quantités infimes de virus. L'apparition il y a quelques années d'une technique révolutionnaire qui permet d'amplifier de façon exponentielle une portion de matériel génétique a donc fourni une arme supplémentaire aux chercheurs. Cette technique, la réaction de polymérisation en chaîne (polymerase chain reaction; $P C R)$, a récemment permis de mettre en cause le virus T-lymphotrope humain de type 1 (HTLV-1) dans la sclérose en plaques [24]. Très sensible, la technique de PCR peut être responsable de faux résultats positifs. Plusieurs travaux ont d'ailleurs récemment récusé les résultats impliquant le virus HTLV-1 [25]. Forts de cette expérience malheureuse, les chercheurs ont élaboré une série de procédures expérimentales qui réduisent le problème des faux signaux positifs de la PCR [26]. En mettant à profit ces mesures, nous avons effectué une étude-pilote qui indique pour la première fois la présence de coronavirus humains dans le cerveau de quatre sur onze malades atteints de sclérose en plaques et aucun des onze tissus contrôles [27]. Une étude plus poussée qui mettra aussi à profit l'hybridation in situ et l'immunohistochimie est en cours. De plus, nous avons récemment démontré que diverses cellules du système nerveux humain sont sensibles à l'infection par les coronavirus humains.

Bien que l'implication des virus dans la sclérose en plaques demeure très plausible, les études qui rapportent l'association de ceux-ci à la maladie demeurent controversées et souvent mises en doute car difficiles à reproduire dans d'autres laboratoires. Le Tableau I résume la quinzaine de virus qui ont été associés à la sclérose en plaques au cours des années. Force est de constater qu'il est peu probable qu'un seul virus constitue l'agent causal de la sclérose en plaques mais que plusieurs virus pourraient être des acteurs importants, soit par synergie entre eux et d'autres cofacteurs, soit par un mécanisme commun d'induction des réactions immunitaires anormales de la maladie.

\section{Mécanismes} de démyélinisation

Comment une infection virale pourrait-elle déclencher les processus pathogènes conduisant à la sclérose en plaques? Si l'on accepte qu'il s'agit d'une maladie démyélinisante, une infection virale persistante pourrait déclencher cette démyélinisation de façon directe ou indirecte, tel que résumé sur le Tableau II.

L'infection cytopathique des oligodendrocytes, les cellules gliales qui sont responsables de la formation de la gaine de myéline, ou encore la destruction directe de celle-ci par une protéine virale qui aurait une activité enzymatique sont deux possibilités envisageables, la première ayant son parallèle dans le modèle animal du coronavirus murin neurotrope décrit ci-dessus.

Un effet indirect de l'infection virale est plus probable, considérant les difficultés énormes à associer un agent infectieux unique dans la sclérose en plaques. Plusieurs hypothèses s'offrent alors. Les oligodendrocytes infectés pourraient devenir la cible d'une destruction par le système immunitaire. Il est bien connu que, lors d'une infection, les lymphocytes activés de la périphérie peuvent traverser la barrière hémato-encéphalique qui isole normalement le système nerveux. Cette barrière est

\begin{tabular}{|c|}
\hline Tableau I \\
VIRUS ASSOCIÉS \\
A LA SCLÉROSE EN PLAOUES \\
\hline Rougeole \\
Rubéole \\
Oreillons \\
Rage \\
Influenza type C \\
Para-influenza type 1 \\
Respiratoire syncytial \\
Flavivirus \\
Coronavirus \\
T-Iymphotrope type 1 \\
Cytomégalovirus \\
Epstein-Barr \\
Vaccine (variole) \\
Herpes simplex \\
Varicelle \\
\hline
\end{tabular}




\section{RÉFÉRENCES}

23. Murray RS, Brown B, Brian D, Cabirac GF. Detection of coronavirus RNA and antigen in multiple sclerosis brain. Ann Neurol $1992 ; 31: 525-33$.

24. Reddy EP, Sandberg-Wollheim M, Met tus RV, Ray PE, DeFreitas E, Koprowski H. Amplification and molecular cloning of HTLV-1 sequences from DNA of multiple sclerosis patients. Science 1989; 243 : 529-33.

25. Dekaban GA, Rice GPA. Retroviruses and multiple sclerosis. 2. Failure of gene amplification techniques to detect viral sequences unique to the disease. Neurology $1990 ; 40: 12548$.

26. Kwok S, Higuchi R. Avoiding false positives with PCR. Nature 1989 ; 339: 237-8.

27. Stewart JN, Mounir S, Talbot PJ. Human coronavirus gene expression in the brains of multiple sclerosis patients. Virology 1992 ; 191 : 502-5.

28. Poser CM. The pathogenesis of multiple sclerosis. Additional considerations. I Neuro Sci 1993; 115 : S3-15.

29. Wee Yong V, Antel J. Major histocompatibility complex molecules on glial cells. Sem Neurosci 1992; 4: 231-40.

30. Jouvenne P, Mounir S, Stewart JN Richardson CD, Talbot PJ. Sequence analy sis of human coronavirus $229 \mathrm{E}$ mRNAs 4 and 5: evidence for polymorphism and homology with myelin basic protein. Virus Res 1992; 22 : 125-41.

31. Wucherpfennia KW, Strominger JL. Molecular mimicry in T cell-mediated autoimmunity: viral peptides activate human $\mathrm{T}$ cell clones specific for myelin basic protein Cell 1995; 80: 695-705.

32. Hafler DA, Weiner HL. MS : a CNS and systemic autoimmune disease. Immunol Today $1989 ; 10: 104-7$.

33. Reingold SC. Advances in the understanding and treatment of multiple sclerosis. J Neuroimmunol 1993; 44 : 221-4.

34. Weiner HL, Mackin GA, Matsui M, Orav EJ, Khoury SJ, Dawson DM, Hafler DA. Double-blind pilot trial of oral tolerization with myelin antigens in multiple sclerosis. Science 1993 ; 259: 1321-4.

35. The Interferon multiple sclerosis study group. Interferon $\beta-1 \mathrm{~b}$ is effective in relapsing-remitting multiple sclerosis. Neurology

\begin{tabular}{l} 
Tableau II \\
MÉCANISMES POSSIBLES DE DÉMYÉLINISATION INDUITE \\
PAR LES VIRUS \\
\hline 1. Effets viraux directs \\
a. Infection cytopathique des oligodendrocytes \\
b. Destruction de la myéline par des protéines virales \\
2. Effets immunitaires induits par les virus \\
a. Destruction immunitaire des oligodendrocytes infectés \\
b. Sensibilisation aux antigènes de la myéline \\
- Relargage des composants de la myéline \\
- Incorporation de la myéline dans l'enveloppe virale \\
- Nouvelles structures immunogéniques \\
c. Induction d'antigènes d'histocompatibilité \\
d. Mimétisme moléculaire virus-myéline
\end{tabular}

formée des cellules endothéliales qui bordent les vaisseaux sanguins irriguant le cerveau et pourrait même être la cible primaire de la réponse immunitaire observée chez les patients atteints de sclérose en plaques [28]. Une infection des oligodendrocytes pourrait aussi relarguer des composants de la myéline qui ne sont normalement pas présentés au système immunitaire. L'association de protéines virales à des protéines de la myéline pour former de nouvelles structures ou même l'incorporation dans l'enveloppe virale de composants de la myéline lors du bourgeonnement du virus quittant la cellule infectée constituent d'autres hypothèses. Tel que démontré dans le modèle animal du coronavirus murin neurotrope, une infection virale pourrait induire l'expression d'antigènes d'histocompatibilité sur les cellules nerveuses et gliales qui ne les expriment normalement pas [29], ce qui pourrait activer les réponses immunes locales.

Enfin, un mécanisme très attrayant d'induction de l'auto-immunité antimyéline par une infection virale fait intervenir une similitude de séquences protéiques entre des protéines virales et celles de la myéline. Une réponse immunitaire antivirale pourrait donc devenir auto-immune par ce que l'on appelle un phénomène de mimétisme moléculaire. Il reste alors à postuler que les personnes qui développent la sclérose en plaques sont génétiquement prédisposées, entre autres par leur haplotype HLA-DR2, à reconnaître ces séquences similaires. Nous avons récemment mis en évidence un tel mimétisme moléculaire entre une protéine du coronavirus humain et la protéine basique de la myéline (MBP) [8, 30] et avons commencé une étude destinée à vérifier la pertinence de telles analogies avec divers virus dont le coronavirus dans l'étiologie de la sclérose en plaques. Les premiers résultats de cette recherche suggèrent que le système immunitaire de plusieurs patients atteints de la maladie montre des réactions croisées entre un coronavirus humain et la MBP, contrairement à des témoins. Nos résultats sont maintenant appuyés par l'observation récente que des peptides de sept différents virus sont reconnus par des lymphocytes T spécifiques de la MBP [31].

\section{Réponses immunitaires : voies thérapeutiques possibles}

Bien que cette proposition soit encore contestée par certains, la sclérose en plaques semble être une maladie auto-immune dont les antigènes cibles ne sont pas encore identifiés avec certitude mais pourraient être des protéines de la myéline comme la protéine basique de la myéline $[13,32]$. Compte tenu de l'importance cruciale dans le développement de la maladie de la présentation d'autoantigènes au système immunitaire par l'intermédiaire d'antigènes d'histocompatibilité de classe II et des lymphocytes $\mathrm{T}$ auxiliaires $\left(\mathrm{CD}^{+}\right)$, plusieurs traitements possibles sont 
maintenant à l'étude. Trois approches expérimentales sont privilégiées en ce moment: induction d'un état d'anergie, c'est-à-dire nonfonctionnel, des lymphocytes $\mathrm{T}$ autoréactifs par immunisation avec des récepteurs homologues des récepteurs $\mathrm{T}$ impliqués dans la maladie; induction de la tolérance de l'organisme par injection de molécules antagonistes en compétition avec les autoantigènes pour la liaison des antigènes HI A de classe II ; induction de la tolérance à la protéine basique de la myéline par ingestion orale de celle-ci $[33,34]$. Il est encore trop tôt pour conclure quant aux bénéfices possibles de ces traitements expérimentaux.

Un pas important a été franchi récemment lors de la découverte que l'injection d'interféron $\beta$-1b constitue le premier traitement pouvant de façon scientifiquement contrôlée diminuer les attaques de la maladie chez les patients souffrant de la forme cyclique de la sclérose en plaques [35], les travaux se poursuivant très activement pour la forme chronique de la maladie. Parmi ses diverses actions sur le système immunitaire, il est fascinant de noter que l'interféron $\beta$ est un agent antiviral. Les auteurs de l'étude clinique le mentionnent d'ailleurs dans leur discussion des mécanismes d'action possible de ce produit, notant que des études ont précédemment démontré une corrélation entre des infections virales et les exacerbations de la sclérose en plaques [35].

\section{Conclusion}

Même s'il peut être prouvé qu'un ou plusieurs virus sont impliqués dans la sclérose en plaques, il est fort probable que ce ou ces virus ne seront que les agents déclenchants d'une série de phénomènes immunologiques aboutissant à la démyélinisation des fibres nerveuses. D'ailleurs, l'hypothèse virale de la sclérose en plaques ne demeure plausible que si l'on postule qu'il n'existe pas un virus unique causant la maladie. En effet, il serait alors bien surprenant qu'il n'ait pas encore été identifié plus clairement. Il demeure toutefois primordial de continuer à explorer toutes les pistes virales intéressantes qui nous rapprochent d'une meilleure compréhension des causes de la sclérose en plaques. L'implication directe ou indirecte des coronavirus constitue l'exemple le plus récent d'une telle piste qu'il importe de ne pas ignorer. Une collaboration étroite entre neurologues, immunologistes et virologistes devrait permettre de progresser vers la prévention et la guérison de la sclérose en plaques. En chemin, nous acquerrons des informations importantes à la compréhension d'autres maladies neurologiques impliquant peut-être des virus.

Les plus optimistes attendent un déclin de la sclérose en plaques grâce aux programmes de vaccination contre la rougeole, la rubéole et les oreillons. D'autres font remarquer qu'il existe plusieurs précédents où une maladie peut être traitée avant même que sa cause ne soit connue: le scorbut et le choléra en sont deux

\section{TIRÉS À PART}

P. Talbot.

\section{Remerciements} article.

exemples. Il est donc permis d'espérer des progrès de plus en plus rapides

Les travaux effectués dans mon laboratoire sont subventionnés par le Conseil de recherches médicales du Canada, le Fonds de la recherche en santé du Québec et la Société canadienne de la sclérose en plaques. Je remercie Francine Lambert pour son savoir-faire technique, incluant la préparation de la figure 1, qui a été réalisée en microscopie électronique par Robert Alain. Ma gratitude va aussi à Patricia Jouvenne et Nathalie Arbour, étudiantes de doctorat, Geneviève Côté, Fanny Chagnon, Sophie Erandé et Janet N. Stewart, étudiantes de maîtrise, Aurelio Bonavia et JeanSébastien Poquette, étudiants de maîtrise, Samir Mounir, stagiaire post-doctoral et France Ouellet, agent de recherche, qui ont participé directement aux travaux mentionnés dans cet

\section{Summary \\ Implication of viruses in multiple sclerosis}

Multiple sclerosis (MS) is an inflammatory neurological disease that results from an immune attack and destruction of the myelin sheath that normally surrounds nerve fibers. This chronic and recurrent demyelination perturbs the normal flow of nerve impulses and leads to a debilitating disease that affects about $0.2 \%$ of young adults in high-incidence areas such as Canada, making it the most prevalent neurological disease in this age group. Despite a still mysterious etiology, it is clear that the development of MS involves both genetic factors such as immune response genes HLA-DR2, and environmental factors, most likely infectious agents. About fifteen different viruses have been associated with MS over the years, but not a single agent has yet emerged as the strongest candidate. Coronaviruses are one of the most recent viral agents reported in association with MS, but more research is needed to strengthen their possible involvement. Unfortunately, coronaviruses have yet to attract the attention of the medical community, having been so far only implicated in relatively benign respiratory infections of the common cold type. On the other hand, coronaviruses in mice have provided for several years one of the best animal models of a viral disease that resembles human MS. The viral hypothesis for MS remains highly reasonable but is unlikely to involve only one virus but rather several viruses that could initiate by a common mechanism the immune disorders leading to the demyelination of MS. Hence, all viral possibilities and relevant animal models need to be explored in order to gain a better understanding of the mechanisms involved in disease initiation. In parallel, various promising treatments need to be scientifically validated to provide hope to patients while the elucidation of the exact causes of MS slowly proceeds, faster progress rendered possible by a close collaboration between virologists, immunologists and neurologists. 